\title{
Robust Extrapolation Scheme for Fast Estimation of 3D Ising Field Partition Functions: Application to Within-Subject fMRI Data Analysis *
}

\author{
Laurent Risser ${ }^{1,2,3}$, Thomas Vincent ${ }^{1,2}$, Philippe Ciuciu ${ }^{1,2}$, and Jérôme Idier ${ }^{3}$ \\ 1 NeuroSpin/CEA, 91191 Gif-sur-Yvette, France \\ 2 IFR 49, Institut d'Imagerie Neurofonctionnelle, Paris, France \\ 3 IRCCyN/CNRS, 1 rue de la Noë 44300 Nantes, France
}

\begin{abstract}
In this paper, we present a fast numerical scheme to estimate Partition Functions (PF) of 3D Ising fields. Our strategy is applied to the context of the joint detection-estimation of brain activity from functional Magnetic Resonance Imaging (fMRI) data, where the goal is to automatically recover activated regions and estimate region-dependent hemodynamic filters. For any region, a specific binary Markov random field may embody spatial correlation over the hidden states of the voxels by modeling whether they are activated or not. To make this spatial regularization fully adaptive, our approach is first based upon a classical path-sampling method to approximate a small subset of reference PFs corresponding to prespecified regions. Then, the proposed extrapolation method allows us to approximate the PFs associated with the Ising fields defined over the remaining brain regions. In comparison with preexisting approaches, our method is robust to topological inhomogeneities in the definition of the reference regions. As a result, it strongly alleviates the computational burden and makes spatially adaptive regularization of whole brain fMRI datasets feasible.
\end{abstract}

\section{Introduction}

In fMRI, one usually resorts to spatial filtering to enhance the signal-to-noise ratio at the expense of a loss of spatial resolution. A more challenging approach works on the unsmoothed data by introducing some prior knowledge on the sought spatial structures through for instance local interaction models such as Markov Random Fields (MRFs). Discrete MRFs, which have been used in segmentation and clustering, typically involve a set of hyper-parameters: the smaller this number the less complex the patterns modelled by the corresponding MRF. For instance, a single temperature level controls the amount of spatial correlation in symmetric Ising fields. In the considered fMRI application [1], such Ising fields are hidden since the activation detection process is modelled a priori through a

\footnotetext{
^ We are thankful to Région Ile de France for funding.
} 
two-class Spatial Mixture Model (SMM). Moreover, their definition varies within a brain parcellation that segregates the $4 \mathrm{D}$ data into $\Gamma$ functionally homogeneous non-regular parcels $(\Gamma \approx 500)$ in order to take the regional fluctuations of the Hemodynamic Filter (HF) into account. The Joint Detection-Estimation (JDE) analysis thus consists of a parcel-based analysis where $\Gamma$ independent HFs as well as stimulus-dependent SMMs are identified in order to detect the regions involved in the paradigm and their underlying dynamics (see Section 21). Therefore, several hundreds of temperature levels have to be estimated making a hand-tuning procedure unrealistic. Moreover, since the optimal setting of such a parameter may be different when considering different parcels, all parameters cannot be fixed to the same value. The purpose of this paper is then to provide an unsupervised scheme for adapting the amount of spatial regularization in several hundreds of Ising fields with different topological configurations.

For a single Ising field, estimating the temperature hyper-parameter requires a precise estimation of its Partition Function (PF) ${ }^{1}$ since the exact PF computation is intractable (see Section 3). This estimation can be achieved using importance sampling techniques [2,3]. Recently, alternative strategies have been introduced in the literature 4,5. In short, they resort to continuous MRFs and logistic transform to build up an approximation that maps continuous weights to the binary latent variables in a mixture model. While efficient, these approaches require the setting of an additional scale parameter that controls the accuracy of the approximation and whose optimal value may depend on the application. Moreover, these approximation techniques have been validated only for MRFs defined over regular grids. Hence, we propose in Section 3 a fast and robust extrapolation technique to multiple PF estimation. In comparison to preexisting approaches, our method is robust against grid inhomogeneities and efficient irrespective of the parcel configurations. Validation on real fMRI data is perfomed in Section 4. In Section 5. we discuss the pros and cons of our approach, which has applications in image processing beyond the fMRI context.

\section{Joint Detection-Estimation of Brain Activity in fMRI}

The JDE framework proposed [1,6] relies on a prior parcellation of the brain into $\mathcal{P}=\left(\mathcal{P}_{\gamma}\right)_{\gamma=1: \Gamma}$ functionally homogeneous and connected parcels [7. Every parcel $\mathcal{P}_{\gamma}$ comprising voxels $\left(V_{j}\right)_{j=1: J}$ is characterized by a region-based model of the BOLD signal, which consists in estimating a single HF shape $\boldsymbol{h}_{\gamma}$ whatever the number of experimental conditions involved in the paradigm.

In a given parcel $\mathcal{P}_{\gamma}$, voxel-dependent and stimulus-induced fluctuations of the BOLD signal are encoded by spatially varying magnitudes a $=\left(a_{j}^{m}\right)_{j=1: J}^{m=1: M}$, where $m$ stands for the stimulus type index. The fMRI time course measured in voxel $V_{j} \in \mathcal{P}_{\gamma}$ then reads: $\boldsymbol{y}_{j}=\sum_{m=1}^{M} a_{j}^{m} \boldsymbol{x}^{m} \star \boldsymbol{h}_{\gamma}+\boldsymbol{b}_{j}$, where $\boldsymbol{x}^{m}$ stands for the $m^{\text {th }}$ binary stimuli vector and $\boldsymbol{b}_{j}$ stands for the serially correlated noise component [1, 6]. Within the Bayesian framework, prior probability density

${ }^{1}$ The normalizing constant that makes the MRF measure of unit mass over its domain. 
functions (pdfs) are introduced on every sought object i.e., (a, $\left.\boldsymbol{h}_{\gamma}\right)$ [1]. Spatial Gaussian mixture models are expressed on a through the introduction of hidden variables $\mathbb{q}=\left(\boldsymbol{q}_{j}^{m}\right)_{j=1: J}^{m=1: M}$ that encode whether voxel $V_{j}$ is activating in response to stimulus $m\left(q_{j}^{m}=1\right)$ or not $\left(q_{j}^{m}=0\right)$. Hence, stimulus-dependent hidden symmetric Ising fields are introduced on these states:

$$
\begin{aligned}
\operatorname{Pr}\left(\boldsymbol{q}^{m} \mid \beta_{m}\right) & =Z\left(\beta_{m}\right)^{-1} \exp \left(\beta_{m} U\left(\boldsymbol{q}^{m}\right)\right), \\
\text { with } \quad Z\left(\beta_{m}\right) & =\sum_{\boldsymbol{q}^{m} \in\{0,1\}^{n}} \exp \left(\beta_{m} U\left(\boldsymbol{q}^{m}\right)\right)
\end{aligned}
$$

and $U\left(\boldsymbol{q}^{m}\right)=\sum_{i \sim j} I\left(q_{i}^{m}=q_{j}^{m}\right)$ is the global "negative energy" where $I(A)=1$ whenever $A$ is true and 0 otherwise. The parameter $\beta_{m} \geq 0$ controls the amount of spatial correlation between the components of $\boldsymbol{q}^{m}$ according to the grid $\mathcal{G}$, while $Z\left(\beta_{m}\right)$ defines the partition function. The global prior SMM then reads:

$$
p\left(\mathrm{a} \mid \Theta_{\mathrm{a}}\right)=\prod_{m} p\left(\boldsymbol{a}_{m} \mid \boldsymbol{\theta}_{m}\right)=\prod_{m}\left\{\sum_{\boldsymbol{q}^{m}}\left[\prod_{j} f\left(a_{j}^{m} \mid q_{j}^{m}, \boldsymbol{\theta}_{m}\right)\right] \operatorname{Pr}\left(\boldsymbol{q}^{m} \mid \boldsymbol{\beta}_{m}\right)\right\}
$$

where $f\left(a_{j}^{m} \mid q_{j}^{m}=i\right) \sim \mathcal{N}\left(\mu_{i, m}, v_{i, m}\right)$. Parameters $\mu_{i, m}$ and $v_{i, m}$ define the prior mean and variance of class $i=0,1$, respectively for the stimulus type $m$. Let the set $\boldsymbol{\theta}_{m}$ gather mixture parameters $\left\{\mu_{0, m}, \mu_{1, m}, v_{0, m}, v_{1, m}, \beta_{m}\right\}$. Samples of the full posterior pdf $p\left(\boldsymbol{h}_{\gamma}\right.$, a, $\left., \mathbb{q}, \boldsymbol{\Theta} \mid \mathbb{y}\right)$ are simulated using a Gibbs sampler algorithm and posterior mean estimates are then computed from these samples. Here, we specifically deal with the sampling of parameter $\beta_{m}$, which is achieved using a symmetric random walk Metropolis-Hastings step: at iteration $k$, a candidate $\beta_{m}^{(k+1 / 2)} \sim \mathcal{N}\left(\beta_{m}^{(k)}, \sigma_{\epsilon}^{2}\right)$ is generated. It is accepted (i.e., $\beta_{m}^{(k+1)}=\beta_{m}^{(k+1 / 2)}$ ) with probability: $\alpha\left(\beta_{m}^{(k)} \rightarrow \beta_{m}^{(k+1 / 2)}\right)=\min \left(1, A_{k, k+1 / 2}\right)$, where the acceptation ratio $A_{k, k+1 / 2}$ follows from Eq. (1):

$$
\begin{aligned}
A_{k, k+1 / 2} & =\frac{p\left(\beta_{m}^{(k+1 / 2)} \mid \boldsymbol{q}_{m}^{(k)}\right)}{p\left(\beta_{m}^{(k)} \mid \boldsymbol{q}_{m}^{(k)}\right)}=\frac{p\left(\boldsymbol{q}_{m}^{(k)} \mid \beta_{m}^{(k+1 / 2)}\right) p\left(\beta_{m}^{(k+1 / 2)}\right)}{p\left(\boldsymbol{q}_{m}^{(k)} \mid \beta_{m}^{(k)}\right) p\left(\beta_{m}^{(k)}\right)} \\
& =\frac{Z\left(\beta_{m}^{(k)}\right)}{Z\left(\beta_{m}^{(k+1 / 2)}\right)} \exp \left(\left(\beta_{m}^{(k+1 / 2)}-\beta_{m}^{(k)}\right) U\left(\boldsymbol{q}_{m}^{(k)}\right)\right),
\end{aligned}
$$

using Bayes' rule and considering a uniform prior for $\beta_{m}$. The $\beta_{m}$ sampling step then requires to estimate ratios of $Z($.$) or log-PF differences for all \mathcal{P}_{\gamma}$ parcels prior to exploring the full posterior pdf. This motivates the need for developing fast approximation techniques of these constants.

\section{Ising Field PF Estimation}

\subsection{Single PF Estimation}

Exact evaluation of $Z(\cdot)$ in a reasonable amount of time is impossible except on tiny grids. Robust and fast estimation of $Z(\beta)$ is thus a key issue for numerous $3 \mathrm{D}$ imaging problems involving Ising models and more generally discrete MRFs. 
Path-sampling is an extension of importance sampling for estimating ratios of normalizing constants, by considering series of easy-to-sample unormalized intermediate densities. Such a strategy was proven efficient to tabulate the PF for the Ising case; see 8 for details. Time dedicated to a single PF estimation using this method is acceptable but becomes penalizing when numerous PFs need to be estimated as required when dealing with several hundreds of grids of variable size and shape. Since this typical situation occurs in our fMRI application, a fast compromise consists in resorting to path-sampling to get log-scale estimates $\left(\log \widehat{Z}_{\mathcal{G}_{p}}(\beta)\right)_{p=1: P}$ for a small subset of reference grids $\left(\mathcal{G}_{p}\right)_{p=1: P}$ and then in using extrapolation formulas to obtain $\log \widetilde{Z}_{\mathcal{T}}(\beta)$ for the large remaining set of brain parcels to be analyzed, referenced here by a test grid $\mathcal{T}$.

\subsection{Multiple PF Estimation}

In [8], the authors address linear regression on $\left(\log \widehat{Z}_{\mathcal{G}_{p}}(\beta)\right)_{p=1: P}$ as a function of the number of cliques in the grids $\left(\mathcal{G}_{p}\right)_{p=1: P}$. Estimates $\log \widetilde{Z}_{\mathcal{T}}(\beta)$ are then linearly computed from the regression coefficients and the number of cliques in $\mathcal{T}$. A bilinear extension of this technique, which also accounts for the number of sites in the grid, can be thought of to estimate log-PFs in small and non-regular grids 2 such as those appearing in our fMRI application. However, it can be shown that the accuracy of regression-based techniques strongly depends on the homogeneity and the number of reference grids: the more inhomogeneous the reference set, the larger the approximation error. These reasons motivate the development of more reliable approaches regarding the reference PFs.

\subsection{Proposed PF Extrapolation Technique}

The appropriate reference grid is selected by means of a similarity measure and the accuracy of the approximation is controlled by an error criterion. Our algorithm proceeds in two steps: 1) Akin to [8, reference PFs $\widehat{Z}_{\mathcal{G}_{p}}\left(\beta_{k}\right)$ are estimated using path-sampling. The topological configurations of the reference grids $\left(\mathcal{G}_{p}\right)_{p=1: P}$ can now be inhomogeneous to cover a maximum of grid configurations that may occur in further PF estimations. 2) For any test grid $\mathcal{T}, \log Z_{\mathcal{T}}$ is approximated using a single reference log-PF estimate out of $\left(\log \widehat{Z}_{\mathcal{T}_{p}}(\beta)\right)_{p=1: P}$.

Let $r_{\mathcal{T}}=\sigma_{n, \mathcal{T}} / \mu_{n, \mathcal{T}}$ be a measure of grid homogeneity where $\mu_{n, \mathcal{T}}$ and $\sigma_{n, \mathcal{T}}$ respectively provide the average number of neighbors per site over $\mathcal{T}$ and the corresponding standard deviation. The smaller $r_{\mathcal{T}}$ the more regular $\mathcal{T}$. Using this criterion, our similarity measure is defined by $\mathcal{L}_{\mathcal{T}}\left(\mathcal{G}_{p}\right)=\left\|r_{\mathcal{T}}-r_{\mathcal{G}_{p}}\right\|^{2}$, which helps us choose the closest reference grid $\mathcal{G}_{\text {ref }}$ to $\mathcal{T}$ in combination with the approximation error criterion $\mathcal{A}_{\mathcal{T}}\left(\beta, \mathcal{G}_{p}\right)$ given by:

$$
\mathcal{A}_{\mathcal{T}}\left(\beta, \mathcal{G}_{p}\right)=\left\|\log Z_{\mathcal{T}}(\beta)-\log \widetilde{Z}_{\mathcal{T}}\left(\beta, \mathcal{G}_{p}\right)\right\|^{2} /\left\|\log Z_{\mathcal{T}}(\beta)\right\|^{2}
$$

where $\log \widetilde{Z}_{\mathcal{T}}\left(\beta, \mathcal{G}_{p}\right)=\left(c_{\mathcal{T}}\left(\log \widehat{Z}_{\mathcal{G}_{p}}(\beta)-\log 2\right) / c_{\mathcal{G}_{p}}+\log 2\right)$.

${ }^{2}$ Here, non-regular grids make reference to regular lattices combined with non-straight boundaries. 


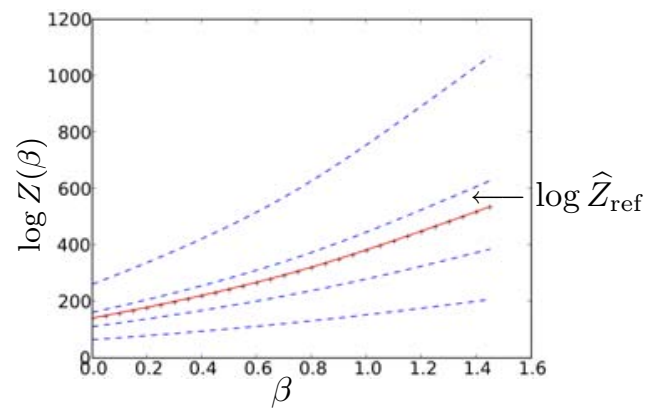

Fig. 1. The blue curves correspond to path-sampling estimates of the reference log-PFs $\log \widehat{Z}_{\mathcal{G}_{p}}\left(\beta_{k}\right), p=1: 4$. The red curve defines the ground truth (path-sampled $\log \widehat{Z}_{\mathcal{T}}$ ). Our extrapolation method provides the crossed-line $\left(x_{-}\right) \log$-PF estimate $\log \widetilde{Z}_{\mathcal{T}}$.

Here, $\left(c_{\mathcal{T}}, c_{\mathcal{G}_{p}}\right)$ and $\left(s_{\mathcal{T}}, s_{\mathcal{G}_{p}}\right)$ are the number of cliques and sites of the Ising fields defined over $\mathcal{T}$ and $\mathcal{G}_{p} . \widetilde{Z}_{\mathcal{T}}\left(\beta, \mathcal{G}_{p}\right)$ corresponds to the $Z_{\mathcal{T}}(\beta)$ estimate computed using $\mathcal{G}_{p}$. Our extrapolation formula (3) is built up according to two principles directly derived from the PF definition: $i$.) an unbiased asymptotic approximation error $\left(\lim _{\beta \rightarrow+\infty} \mathcal{A}_{\mathcal{T}}\left(\beta, \mathcal{G}_{p}\right)=0\right)$ and ii.) an exact approximation of the first derivative of $\log Z_{\mathcal{T}}(\beta)$ for $\beta \rightarrow 0^{+}$. Interestingly, our extrapolation technique makes also possible the analytical computation of the approximation error $\mathcal{A}\left(\beta, \mathcal{G}_{p}\right)$ at $\beta=0$, a value for which the error is maximal when $\mathcal{L}_{\mathcal{T}}\left(\mathcal{G}_{p}\right)$ is sufficiently low. This property were empirically verified on more than 300 fields simulated over regular and non-regular grids. The reference grid is then exhibited using a min-max principle, which consists in minimizing the error $\mathcal{A}\left(0, \mathcal{G}_{p}\right)$ wrt all reference grids $\left(\mathcal{G}_{p}\right)_{p=1: P}$ when the homogeneity of $\mathcal{T}$ and $\mathcal{G}_{p}$ is similar:

$$
\begin{gathered}
\mathcal{G}_{\text {ref }}=\underset{\left(\mathcal{G}_{p}\right)_{p=1: P}}{\arg \min } \mathcal{A}_{\mathcal{T}}\left(0, \mathcal{G}_{p}\right) \quad \text { subject to } \quad \mathcal{L}_{\mathcal{T}}\left(\mathcal{G}_{p}\right) \leq \epsilon \\
\text { with } \quad \mathcal{A}_{\mathcal{T}}\left(0, \mathcal{G}_{p}\right) \triangleq\left\|\left(s_{\mathcal{T}}-1\right)-c_{\mathcal{T}}\left(s_{\mathcal{G}_{p}}-1\right) / c_{\mathcal{G}_{p}}\right\|^{2} / s_{\mathcal{T}}^{2}
\end{gathered}
$$

where $\epsilon$ is positive threshold fixed by hand 3 . In practice, we first compute $\mathcal{L}_{\mathcal{T}}\left(\mathcal{G}_{p}\right)$ and $\mathcal{A}_{\mathcal{T}}\left(0, \mathcal{G}_{p}\right), \forall \mathcal{G}_{p}$ and only keep the subset $\mathcal{S}$ of reference grids for which the constraint $\mathcal{L}_{\mathcal{T}}\left(\mathcal{G}_{p}\right) \leq \epsilon$ is fulfilled and $\mathcal{A}_{\mathcal{T}}\left(0, \mathcal{G}_{p}\right)$ is below another threshold (typically $4 \%$ ). If $\mathcal{S}$ is empty, $\log Z_{\mathcal{T}}(\beta)$ is estimated using path-sampling. Otherwise, in a second stage, $\mathcal{G}_{\text {ref }}$ is exhibited from $\mathcal{S}$ as the minimizer of $\mathcal{A}_{\mathcal{T}}(0, \mathcal{S})$ and the $\log$-PF estimate in $\mathcal{T}$ is thus given by $\log \widetilde{Z}_{\mathcal{T}}\left(\beta, \mathcal{G}_{\text {ref }}\right)$ according to Eq. (3).

Our method is illustrated in Fig. 1 with $P=4$ : by comparing the distance between the blue and red curves at $\beta=0$, it appears that $\log \widehat{Z}_{\text {ref }}$ is the closest curve above the path-sampled red curve $\log \widehat{Z}_{\mathcal{T}}$ considered here as the ground truth. As shown in Fig. 1, our extrapolation $\log \widetilde{Z}_{\mathcal{T}}$ is superimposed on $\log \widehat{Z}_{\mathcal{T}}$.

$\overline{{ }^{3} \text { We used } \epsilon}=0.02$ in our experiments. 


\section{Results}

\subsection{Simulated Ising Fields}

For validation purpose, we compared log-PF estimates computed using our extrapolation technique with the linear and bilinear alternatives proposed in 8 . Here, the ground truth was given by the PF estimates computed using pathsampling. Reference and test grids are either regular or irregular. Each subset of reference or test grids contains at least 30 grids whose number of voxels varied between $5^{3}$ and $16^{3}$. Irregular grids were stochastically generated as a function of a regularization level $\alpha$ taking its values between 0.2 (highly irregular) and 0.5 (almost regular). All tests were performed on 3D Ising fields defined using a 6 -connectivity system. Percentages of approximation errors are shown in Table1.

Table 1. Mean maximal approximation errors (given in \%) over regular and irregular test grids reported for the linear, bilinear and extrapolation techniques

\begin{tabular}{|c|c|c|c|c|c|c|}
\hline \multirow{2}{*}{\multicolumn{2}{|c|}{ Test grid }} & \multicolumn{5}{|c|}{$\begin{array}{c}\text { Scheme / Reference grid } \\
\mathrm{E}=\text { Extrapolation, } \mathrm{B}=\text { bilinear, } \mathrm{L}=\text { linear } / \mathrm{R}=\text { regular, } \mathrm{I}=\text { irregular }\end{array}$} \\
\hline & & $\mathrm{E} / \mathrm{I} \& \mathrm{R}$ & $\mathrm{B} / \mathrm{I}$ & $\mathrm{B} / \mathrm{R}$ & $\mathrm{L} / \mathrm{I}$ & $\mathrm{L} / \mathrm{R}$ \\
\hline \multirow{3}{*}{ 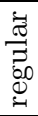 } & small & 0.639 & 3.84 & 66.3 & 93.0 & 2728 \\
\hline & medium & 2.77 & 0.991 & 2.17 & 6.37 & 49.5 \\
\hline & large & 3.68 & 1.31 & 2.48 & 7.18 & 19.4 \\
\hline \multirow{4}{*}{ 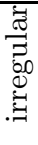 } & $\alpha=0.2$ & 0.375 & 1.29 & 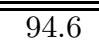 & 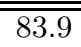 & 3270 \\
\hline & $\alpha=0.3$ & 0.281 & 0.784 & 2.91 & 18.3 & 219 \\
\hline & $\alpha=0.4$ & 0.621 & 0.264 & 3.23 & 8.28 & 34.8 \\
\hline & $\alpha=0.5$ & 0.693 & 1.27 & 1.96 & 1.52 & 34.2 \\
\hline
\end{tabular}

The bilinear and extrapolation methods clearly outperform the linear one. Moreover, as shown in col. (B/ R) and rows (regular small and irregular, $\alpha=0.2$ ), the bilinear method provides inaccurate estimates when there are strong topological differences between the reference and test grids. The regular reference grids are actually composed of large grids with cubic, planar and curvilinar shapes whereas those lying in (regular small) and (irregular $\alpha=0.2$ ) are very small and highly sparse. In this case, our extrapolation method detects such differences and still succeeds in providing reliable log-PF estimates. While the linear/bilinear methods take all reference grids into consideration to derive a log-PF approximation, our approach computes a log-PF estimate using the most appropriate reference grid. Hence, the larger the set of reference grids the more accurate our extrapolation method becomes. This explains why the reference subsets are successfully mixed in the proposed approach, as shown in the first column of Table1. Interestingly, when both the reference and test grids are non-regular the bilinear method may provide a competitive alternative to our extrapolation technique.

\subsection{Real fMRI Dataset}

Our extrapolation algorithm was applied to the spatially adaptative regularization of real fMRI data recorded during an event-related experiment designed to 

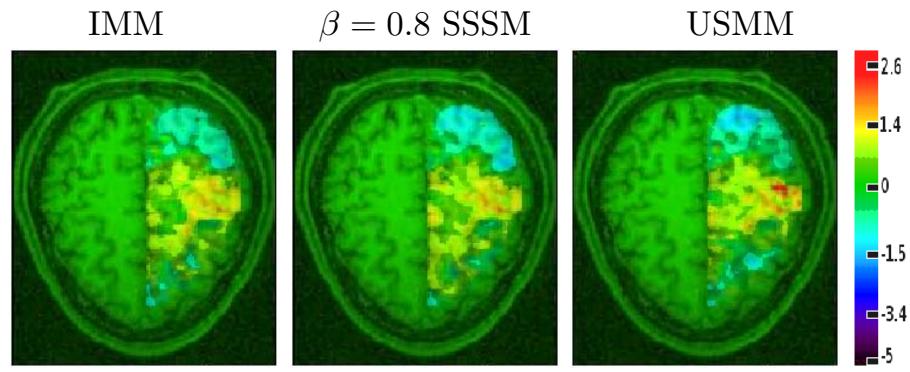

Fig. 2. From left to right: comparison of the IMM, SSMM and USMM models wrt the estimated normalized constrat maps: left vs. right auditory clicks: $\widehat{\boldsymbol{a}}^{\mathrm{LAC}}-\widehat{\boldsymbol{a}}^{\mathrm{RAC}}$

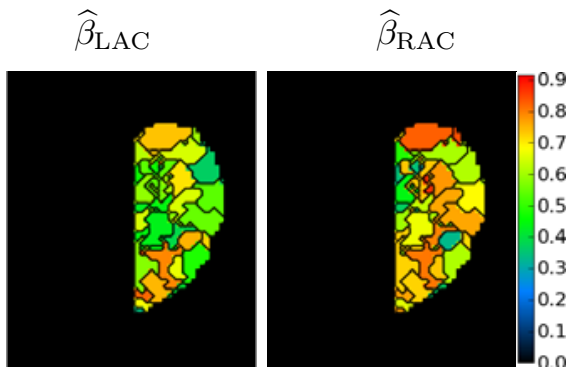

Fig. 3. Left: $\widehat{\beta}_{\text {LAC }}$ parcel-dependent map computed for the LAC condition. Right: $\widehat{\beta}_{\mathrm{RAC}}$ parcel-dependent map computed for the RAC condition.

quickly map main sensory cortices (auditory, visual, motor) as well as higher cognitive functions (reading, computation). Acquisition consisted of a single session (125 scans, $\mathrm{TR}=2.4 \mathrm{~s}, 64 \times 64 \times 32$ volumes). The paradigm comprised sixty stimuli, declined in 10 experimental conditions.

We compare three versions of the JDE procedure: Independent Mixture Models (IMM), Supervised SMM (SSMM, $\beta=0.8$ ) and unsupervised SMM (USMM), in order to assess the impact of the adaptive spatial correlation model. Fig. 2 shows normalized contrast maps $\left(\widehat{\boldsymbol{a}}^{\mathrm{LAC}}-\widehat{\boldsymbol{a}}^{\mathrm{RAC}}\right)$ of auditory induced left versus right clicks (LAC vs RAS). As expected, the activations lie in the contralateral right motor cortex. Here, only USMM is more sensitive illustrating therefore the advantage of an adaptive spatial correlation model. Indeed, estimated $\widehat{\beta}^{\mathrm{PM}}$ with USMM for the left auditory click was 0.56 so that the supervised setting of SSMM with $\beta=0.8$ leads to too much correlation and less sensitive results.

Interestingly, Fig. 3 depicts the parcel-dependent maps of the $\widehat{\beta}^{\mathrm{PM}}$ estimates for the RAC and LAC experimental conditions. The gain in sensitivity in the USMM contrast map $\left(\widehat{\boldsymbol{a}}^{\mathrm{LAC}}-\widehat{\boldsymbol{a}}^{\mathrm{RAC}}\right)$ clearly results from a difference in the amount of spatial regularization introduced between the two conditions involved in the contrast. A lower regularization level is estimated $\left(\widehat{\beta}_{\mathrm{LAC}} \approx 0.5 \mathrm{vs} . \widehat{\beta}_{\mathrm{RAC}} \approx\right.$ $0.75)$ in parcels located in the right motor cortex since the BOLD signal is 
stronger for the LAC condition than for the RAC one in these regions. Hence, the noise induced by the RAC condition is smoothed using a large amount of spatial regularization.

On these real fMRI data, our extrapolation scheme provides log-PFs estimate for a brain parcellation $\left(\mathcal{P}_{\gamma}\right)_{\gamma=1: 300}$ and $\left(\mathcal{G}_{p}\right)_{p=1: 50}$ reference grids. In terms of computational complexity, these log-PF estimates were computed in about ten seconds, a very appealing approach in comparison to path-sampling, which requires about one hour for estimating all log-PF estimates for a negligable gain in accuracy (less than 3\%). Finally, we did not observe any significant difference between the USSM effect maps derived using path sampling and our extrapolation scheme (results not shown).

\section{Discussion and Conclusion}

In order to make spatially adaptive regularization feasible, the considered joint detection-estimation of brain activity from unsmoothed fMRI data requires a reliable and fast estimation of 3D Ising field partition function. To this end, an extrapolation algorithm that exploits pre-computed path-sampled log-PF estimates on reference grids has been proposed. The approximation error is controlled so that the approach defaults to the robust path-sampled PF estimates if no suitable reference candidate is found. Obviously, efficiency is conditionned by the number of reference grids, and more importantly by their similarity to the topologies encountered in the conducted analysis. In practice, about ten additional problem-specific reference grids are enough to provide good PF estimates.

Using our fast extrapolation technique, the computational burden remains acceptable since whole brain data analysis at the subject level takes about 1 h30. The application to real fMRI data showed a gain in statistical sensitivity for the unsupervised version. In order to be properly validated, these promising within-subject results have to be confirmed in a group-level analysis.

\section{References}

1. Vincent, T., Ciuciu, P., Idier, J.: Spatial mixture modelling for the joint detectionestimation of brain activity in fMRI. In: 32th Proc. IEEE ICASSP, Honolulu, Hawaii, vol. I, pp. 325-328 (2007)

2. Meng, X., Wong, W.: Simulating ratios of normalizing constants via a simple identity: a theoretical exploration. Statistica Sinica 6, 831-860 (1996)

3. Gelman, A., Meng, X.L.: Simulating normalizing constants: from importance sampling to bridge sampling to path sampling. Statistical Science 13, 163-185 (1998)

4. Fernández, S., Green, P.J.: Modelling spatially correlated data via mixtures: a Bayesian approach. J. R. Statist. Soc. B 64, 805-826 (2002)

5. Woolrich, M., Behrens, T., Beckmann, C., Smith, S.: Mixture models with adaptive spatial regularization for segmentation with an application to fMRI data. IEEE Trans. Med. Imag. 24, 1-11 (2005) 
6. Makni, S., Idier, J., Vincent, T., Thirion, B., Dehaene-Lambertz, G., Ciuciu, P.: A fully Bayesian approach to the parcel-based detection-estimation of brain activity in fMRI. Neuroimage 41, 941-969 (2008)

7. Thirion, B., Flandin, G., Pinel, P., Roche, A., Ciuciu, P., Poline, J.B.: Dealing with the shortcomings of spatial normalization: Multi-subject parcellation of fMRI datasets. Hum. Brain Mapp. 27, 678-693 (2006)

8. Trillon, A., Idier, J., Peureux, P.: Unsupervised Bayesian 3D reconstruction for nondestructive evaluation using gammagraphy. In: EUSIPCO, Suisse (2008) 